\title{
Superjective Ontology: A Post-Cartesian Tool for the Near-Death Experience
}

\author{
A. Ashanen Carmen, B.Sc. \\ Edinburgh, Scotland
}

ABSTRACT: This study questions the validity of subjective and objective categories, and suggests a new approach, which I call "superjective ontology," as a tool for resolving archaic difficulties. In the first section, I present evidence that a deeply ingrained problem of "ontological disparity" really does exist, along with the extent to which this conditions our thought patterns. I then summarize monism and dualism, and present the core thesis advancing the argument from superjective ontology. Finally, I suggest evidence that might be sought for the core thesis, with special emphasis on the near-death experience.

KEY WORDS: near-death experience; ontology; philosophy; monism; dualism.

Writing in this Journal, Carl Becker (1995) chided us all for digging out the old bones of the monism/dualism argument and gnawing them over with canine enthusiasm, a project which has been conducted frequently in the arena of near-death studies, with widely varying degrees of success:

The field of near-death studies is not the place for debates about monism and dualism, which has occupied better philosophers than us for millennia, and whose resolution, even if possible, would have no immediate relevance to the survival question. (Becker, 1995, p. 26)

I share some of these reservations, but I am unable to agree with them entirely and tend to distance myself, albeit with the greatest of

Ashanen Carmen, B.Sc., a graduate of Edinburgh University with degrees in evolutionary genetics and in computer science, is a free-lance writer with a life-long interest in philosophy, mythology, spiritual experience, and the meaning of challenging phenomena. Reprint requests should be addressed to Mr. Carmen at Flat 1, 28 Lower Granton Road, Trinity, Edinburgh, EH5 3RS, Scotland, U.K.; e-mail: Ashanen@aol.com. 
respect, from Becker's stance. The idea that the truly major strides to a full understanding of the near-death experience (NDE) can be taken without the appropriate ontological rehabilitation is, in my view, romantic in the extreme. Further, a reorientation of the necessary kind may prove to be one of the most powerful tools we can apply to the problem. The major thrust of this paper will be an attempt at such reorientation.

Those who advocate neurobiological inquiry to deepen our understanding of the NDE need have no fear of this ontology, as neuroscience and our expanding knowledge of the brain will always have an important role to play in these complex and intriguing phenomena. On the other hand, those who advocate a spiritual approach, survival of death, and the idea of an "objectively" existing divine order need not be afraid of it either.

Previously in the Journal, Stuart Twemlow (1994) proposed that reality be defined as a "dialectic" between our subjective and objective categories which, in and of themselves, cannot be said to exist. I have considerable sympathy for this position, and Twemlow and I are in close agreement on a number of points, but $I$ believe that it does not go nearly far enough. As I will argue, even our "dialectic" is a long way from what is "really real," to use Twemlow's term. In addition, I suspect that the universe itself emerges from Twemlow's model stained a little too deeply with the rather distinctive dyes of psychodynamics.

\section{The Case for Ontological Disparity}

I would not be writing this, you would not be reading it, and almost certainly there would be no Journal of Near-Death Studies nor any International Association for Near-Death Studies, no controversial debate about the nature of NDEs, if it were possible simply to look into the brain and see displayed there fully explicated manifestations of physiological processes converting themselves into mental facts by a conversion of the objective into the subjective.

Alas, we are not living in such a world. The gap between observable physiological events and the least of mental phenomena is nontrivial, nor can the two be made to reduce one into the other, despite the fact that our continuing insistence on the unconditional reality of these categories (subjective and objective) is a large part of the problem. In our present day there has arisen an irritating trend for signing away these enormous conundrums with a dismissive wave of the hand and 
a verbal parlor trick. Anyone can "solve" the mind/brain problem or "explain" mentation with a self-tailored definition of consciousness sympathetic to his or her own research interests in psychology, neurology, sociology, linguistics, artificial intelligence, and so on.

It is not quite that simple. At the end of this carrion feast on the purported corpse of our intuitive sense of consciousness, we are still left with the fact that a physical description cannot be made to yield a mental truth. Nor is there any applied science of predicting accurately, let alone deriving, specific mental truths from particular physiological events. What we do have are numerous demonstrable correlations, but correlation is neither explanation nor causation. If it were, we would gain a fantastically efficacious psychotherapy based on the selective stimulation of brain structures and the judicious application of drugs, and we would be able to synthesize with formal demonstration fully mature sequences of creative and cogitative thought processes.

This I mark as the first major symptom of ontological disparity, defined as the jarring discrepancy between the architecture of thought we apply to the world and the implacable resistance to explanation of certain facts to be discovered there. In particular, consciousness is showing no sign of yielding to the subject-object dichotomy or the somewhat paradoxical assertions of materialist monism. The second symptom can be considered the inverse to the first. In the first, facts central to our "normal" experience of consciousness disappear when we pursue them too far into our known (defined) ontological categories. On the opposite side of the coin are the anomalous phenomena that wreak havoc with our safe concepts of subject and object, to say nothing of space, time, death, and causality.

I will not get embroiled here in the question of whether or not these phenomena "actually" exist, as this can easily become a straw man set up for the sole purpose of disallowing any subsequent discussion. It is sufficient to note the existence of persistently repeating accounts of ontological violations. The phenomena of parapsychology are far from being just a random heterogeneous scatter. Many of these phenomena, including NDEs, behave as if they belong to a mysterious "overcategory" that is somehow more "real" than our everyday existence.

Of course, it is always possible that we are the victims of a grand deception or our own allegedly inexhaustible resource for misidentification, and this brings me to the third symptom of ontological disparity: namely, the obstreperous refusal to admit that there is any. This tendency to hang on to the idea that physical reality is the only 
possible and authentic existence has deep psychological roots, and is probably the real action of any biologically-based survival instinct. Our drive to find an explanation in terms of familiar ontological categories almost certainly reflects these instincts and the ego's absolute need for self-validation. The subject is complicated, because the categories we hold to at present are precisely those that appear to bestow upon physical existence an unconditional reality, whether or not they are actually true. Biological organisms therefore have a vested interest in upholding them.

However, the quest to discover some magical substance or material surrogate on which to hang an explanation of the paradoxical and the miraculous is by no means limited to the reductionist mindset. The same misidentification of the underlying problem is found among theorists who attempt to account for the NDE, or who foster metaphysical speculations, in pseudo-physically exotic, but ontologically unremedial, terms, such as astral bodies (Muldoon and Carrington, 1929, 1951; Robert Crookall, 1960, 1964, 1980), fourth dimension (Ouspensky, 1931), electromagnetism (Arnette, 1995), holographic frequency domain (Pribram, 1979; Ring, 1980; Talbot, 1991), superluminal nonbaryonic matter (Dutheil and Dutheil, 1990), dark nonbaryonic matter (Kazanis, 1995), wormholes (Arnette, 1992), and so on. I intend no insult to any of these authors, but I also stand firm by my original criticism: nowhere in any of this is the fundamental problem correctly addressed. These claims have their mirror image in the everrenewed urge to reductionism: omnipotent neuralism (SaavedraAguilar and Gómez-Jeria, 1989, 1994; Gómez-Jeria and Madrid-Aliste, 1996; Persinger, 1983; Blackmore and Troscianko, 1989; Blackmore, 1993); cybernetics (Thaler, 1995); birth trauma (Lawson, 1994; Sagan, 1979); personally tweaked "Darwinism" (Thaler, 1996); depersonalization (Noyes and Kletti, 1976); hallucinations (Siegel, 1981), and so on.

On perusing these theories and the dichotomy they make explicit, it becomes obvious what we have been doing, and the revelation is a real jolt to the system. For this reason, I am addressing my argument not to the level of theory, but to the ontological issue on which all our theories take shape and motion. The bewildering array of these explanations is evidence in itself that the eye of the hurricane lies elsewhere. Many of these theories, in both groupings, conceal a diagnostic feature that can best be expressed in the following way: there exists out there somewhere deep in the brain, in the future, or in a romanticized "greater understanding of science," a set of well disguised but thoroughly orthodox data, a pot of gold at the end of the rainbow that, 
when finally disclosed, will explain all manner of mysteries that at present seem implacable. Unfortunately, I have a dark prediction to make about this particular journey over the rainbow, for we will arrive, unlike Dorothy, very much still in Kansas.

As a branch of pseudoscience, scientism (as opposed to true science) reveals itself by a characteristic signature: first, inability to accommodate alternative thought patterns; second, appeal to authority (for example, the abstract "principles of science") instead of the facts observed about the particular phenomenon under investigation; third, persistent and vocal claims that anyone opposed to the declared viewpoint is being "unscientific"; fourth, topological transformation of data until they conform to a preconceived search image; fifth, cloaking of a precarious argument behind a dense curtain of obscure technical terms to impress the uninitiated or intimidate criticism; sixth, obsessive point-by-point rebuttal of criticism; and seventh, an almost pathological lack of humor.

The desiccated seeds of scientistic reductionism and the colorful blooms of alternative science have this fact in common: they are both trapped in the same implicit assumption that our age-old categories of knowledge are "real" and correctly described. It is impossible to overstate the degree to which we are in the grip of this assumption, because it appears to be confirmed by the statistical mass of our everyday mental and sensory experience. If on the other hand our ontological categories are fundamentally misdescribed, and we deny ourselves at every opportunity the necessary remedial action, we are doomed to discover more and ever more unverifiable "correlates" of physiological events and streams of conscious experience. The many neurobiological models may well run to hundreds of pages filled with endlessly fascinating detail, but it still proves maddeningly impossible to derive mental tablature from physical changes by a process of logical formalism, or pierce through to the innermost secrets of the universe. I do not claim to have the answers, but we should not lapse into the error of assuming that because we may not have the ultimate solution, we cannot correctly identify the problem.

\section{Monism and Dualism}

The temptation to deliver a "one-liner" that resolves at a stroke one of the thorniest of philosophical problems ever to have confronted humanity can be very great. A particular phrase employed by many 
people, and which seems to turn up with regularity, is that of mind and brain being "just opposite sides of the one coin." This is one of those statements that boast aspirations to the profound while at the same time being almost completely meaningless. From first principles, the analogy of a coin could be taken as a metaphor for dualism (heads and tails never directly participate in each other's "reality"), monism (there is only one coin), or an integrated one-world philosophy (we perceive only either heads or tails, but what really exists is the coin). In other words, we can endow it with just about any meaning we please.

If mind and matter are to be seen as the opposite faces of this coin, then what we cannot do is say that mind and brain are just like heads and tails of the one truth, so what really exists is the brain, seen one way or the other. Nor can we make this statement and suddenly shift to a position where the brain has mysteriously become the entire coin. Both of these are subtle sleights of hand that deceive us into believing that a problem has been solved, when it actually has not been solved at all.

In each instance we take the partial truth that is the brain in its usual, pre-coin-metaphor sense, declare it to participate with mind in the one truth, and then immediately proceed to describe the outcome again in terms of the favored partial truth, as if it has complete causal power. Our brain in this pre-metaphor sense was always only the head or the tail, not the entire coin, so an unaccountable transformation has appeared in the middle of the process.

Moving to the second case, we do much the same except that mind is entirely sucked into the category of matter. The trouble with this is that the "brain" that emerges is not the same "brain" that went in just a moment before, and bare matter itself has acquired properties to be discerned nowhere in material metaphysics. We can see that the irreducible quality of "being" inherent to consciousness, and the collectively measurable properties of the material world, remain every bit as problematic as they were before, and cannot be derived in terms of each other. The ontological spark gap has not been bridged.

Traditionally, there are three perspectives one can take on the relationship between the experiencing self and the patterns of the world it appears to inhabit. The first of these identifies the perceived forms (material structures) as primary, and the experiencing self as an emergent structure built from those forms. Remove those structures, and the self is removed absolutely. This is materialism, in which mental reality has a secondary existence, and where we have the attempt to stretch the properties of objectified substance to the point 
where it can sustain its essential materialness and yet account for all the phenomena of mind. This is the position taken, consciously or unconsciously, by theorists who interpret NDEs as epiphenomena of chemical processes in the dying brain, who posit divine love in the (causal) terms of endorphins, meaning in terms of temporal lobe excitation, tunnels in terms of the visual cortex, and so on.

The second position is idealism, in which mental reality is primary, and all that we know of a "physical world" (indeed, in the extreme version, all of the physical world) is constructed out of mental realities. Here we have the attempt to stretch the canvas of mind over the realm of matter. Many versions are possible, but I mention here only the two major ramifications in which mental reality resolves to a pure simplicity (in which pattern is a temporary illusion), or else is embodied in an absolute or transcendental order (in which pattern is actual). The first version is the position taken by Buddhism, and the second in various versions by philosophers like George Berkeley (ideas in mind, placed by God), Plato (world of ideal forms), and Immanuel Kant (things unknowable in themselves, if outside our experience).

Finally, there is dualism, in which both mental reality and physical reality are granted independent actual existence, and neither is ultimately reducible to the other. According to René Descartes, there were two fundamental substances created by God for the completeness of the universe. Res extensa (extended substance, or matter) has actual dimension, is capable of division, but expresses no consciousness. Res cogitans (thinking substance) has no actual dimension, cannot therefore be divided, and is the irreducible nature of mind. The two do not mix, and in humans these two categories correspond to body and soul. Unfortunately, Descartes' position required some vehicle of interaction between body and soul, and he selected for this purpose the pineal gland, which in retrospect discloses itself as a poor candidate (Descartes, 1983/1644, 1990/1641, 1994/1637).

Although I will be making no particular efforts to defend dualism, I should point out that the often-repeated charge of dual nature being logically impossible, because the necessary mechanism of interaction would require either a third thing, and thus a swift advance to infinite regression, or else a shared property, in which case they are not truly dual, is far from being an established fact. This objection, and to a large extent dualism itself, is predicated on the idea of "immiscible substances," after the fashion of oil and water. However, we are also familiar with the seemingly obvious fact that there are incommensurate categories, like titillation and temperature, or radioactivity and 


\section{Figure 1 \\ Mutually Exclusive Perceptual Shifts between Separate Perspectives.}

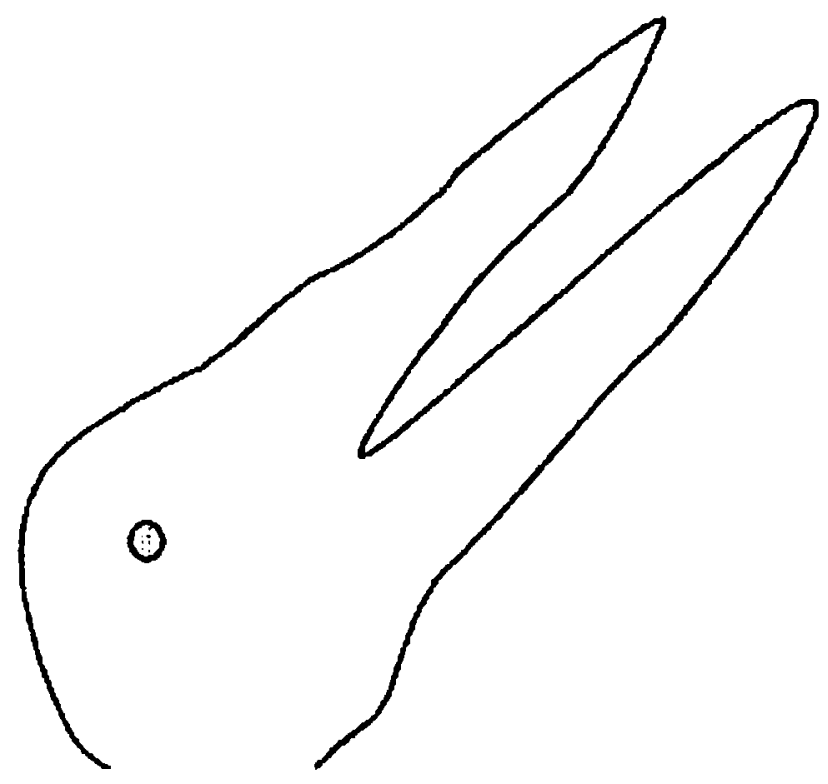

regret. To be willfully absurd about it, we cannot put a fire out with a bucket of sadness. However, even this is a precarious assumption if the phenomena of our common experience are themselves described in incomplete, erroneously assigned, or otherwise conditional categories.

The dualism of separate substances is actually a very crude instrument for analyzing the mind/body question, and is only one possibility among many for conceiving a relationship between the nonidentity of discernibles. Figures 1-3 depict three other ways to address the problem. In Figure 1, we have an alternative representation of the coin argument. However, in this instance it takes the form of mutually 
Figure 2

Transformational Elasticity between Two Separate Entities.

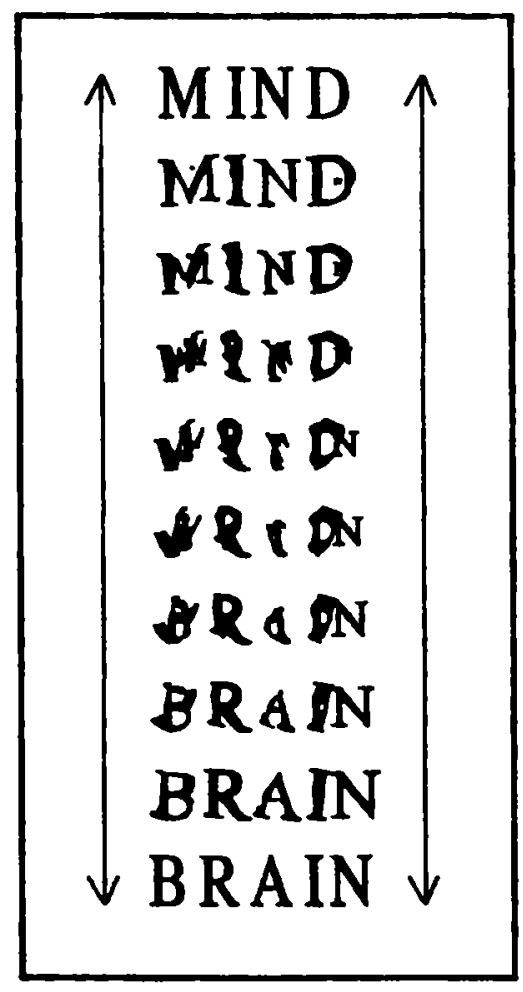

exclusive perceptual shifts: one cannot see the rabbit and the duck simultaneously. If we imagine that "things" do not exist, but only "perspectives," then this becomes an alternative form of dualism.

Figure 2 illustrates the concept of transformational elasticity, missing entirely from substance-based dualism. The important point to grasp here is that this is a conceptual tool, just as the idea of all-ornothing substances is a conceptual tool, and by swapping one tool for the other, the very parameters of the problem begin to change. In this scenario, subject and object are not surgically amputated entities at all, but mere terminal extremes of a versatile transformational process. This understanding will prove important for our purposes.

In Figure 3, yet another way of conceiving the relationship is illustrated. Gottfried Wilhelm Leibniz (1965/1714) proposed the 
Figure 3

Separate Monads Reciprocally Imbedded within Each Other.

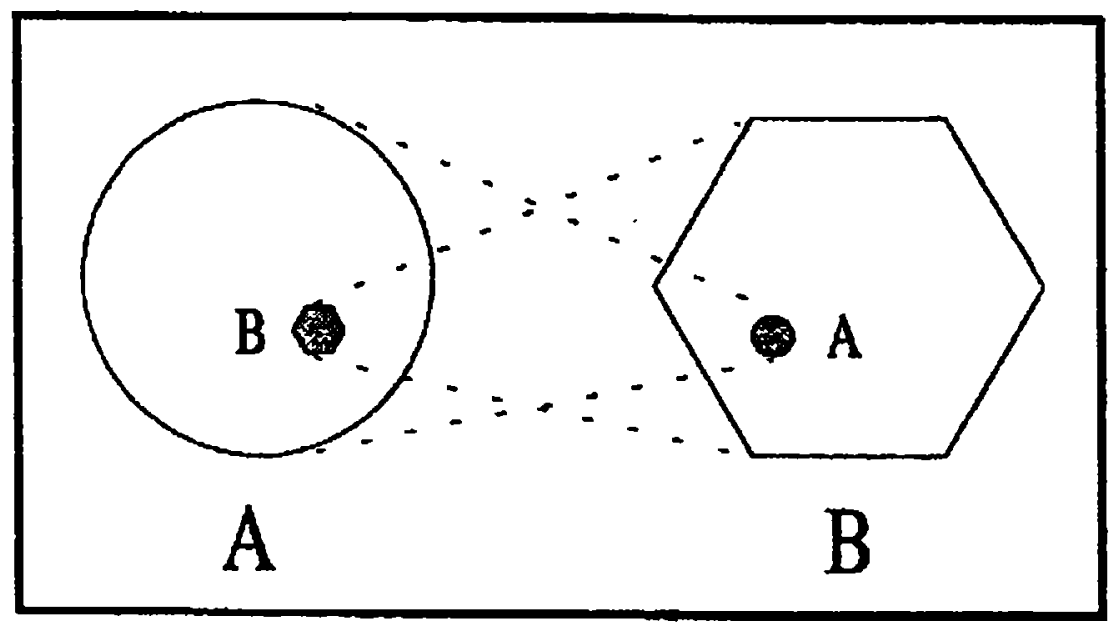

existence of windowless "monads," where each monad reflected from within itself the existence of all other monads. This solved one problem, the unbridgeable gulf of substance dualism, but it created another in the form of an incredible multiplication of monads set in harmony by the will of God. We need not take on the full force of Leibniz's cosmology in order to benefit from the insight of particular relevance to us here. What matters is that the two properties in the system illustrated do not relate to each other as materials separated in space, but contain each other reciprocally in a manner akin to monadic embedding, and where the variation is by strength of influence acting from within. Although it may appear on the diagram that a space separates the two conditions, this is an artifact of presentation and not a true feature of the monad model. When this perspective is combined with that of Figure 2, a very powerful conceptual tool begins to emerge.

\section{Superjective Ontology}

A fundamental concept of our consensus philosophy - the relationship between those categories we call subjective and objective - is being called into question. One of the criticisms most frequently leveled at the idea of a spiritual reality attacks the mysterious location of this other 
world where spiritual things are said to exist. How could something so giant in sheer dimension succeed in keeping itself so well hidden?

I do not believe that it can be an "other world," but this world, the very same universe we inhabit now, at a greater level of assimilation. This is an important point, so let me take a moment to explain what I mean more clearly. Our descriptions of the universe or "what is" achieve various degrees of enclosure, or likelihood of fullness. One of the simplest is to describe the universe as an "object," in the same sense that old boots and mildewed cricket bats are objects. Something of the universe may be caught in this, though perhaps not much. If we wished to make things a little more subtle, we could describe the universe in terms of a "process." The religious idea of a "mind" embodying the universe, or the universe being such a mind, is one attempt at a higher synthesis. This too may capture some truth of the universe, but if we wished to seek out the truth of the universe at its highest or most inclusive level, we may have to go even further than that.

In the form of consciousness human beings possess, everything is split into apparent divisions of subject and object. This, in fact, is the "universe" we inhabit. But suppose that universe (the world we perceive) could be in reality a sub-level perceptual synthesis of the world that really exists, a division into two categories of something that, to itself, exists only in one. Now if that is true, we would be asking entirely the wrong question when we ask, "Where is the spiritual reality?" We could search forever at our own partial level of synthesis and never find it there, because the universe could only ever be an "object" in such a search. The truth we seek exists at a higher level of synthesis.

If we were to conduct this pursuit relentlessly, on our own level, we would find a threshold beyond which the "object" of our attention began to resist all-inclusion. In other words, knowledge concerning the universe (or reality) would refuse to complete itself, such a completion being paradoxical at our level of assimilation. The closer we came to the illusion of totality, the faster a front of ever-escaping knowledge would recede from the observer (ever smaller orders of subatomic particles, ever more elaborate behavior of such particles, paranormal phenomena, elusiveness of consciousness to material observation, impenetrability of time, the Uncertainty Principle, the influence of the observer, and so on).

Fortunately, we now have available a simple visual analogy for the situation I am describing with respect to levels of synthesis, so long as we understand that it is only intended to be a visual aid and is not to be pushed too far. I am alluding to pictures referred to as 
autostereograms or "Magic Eye" images: patterns apparently covered with a random repeating motif, but which yield a complex threedimensional picture if we succeed in relaxing our eyes and making the necessary perceptual shift. No feat of deductive reasoning will enable us to access that reality. It is a cognitive shift that is required, and if we cannot make it, then we are blind with respect to autostereograms. In the world of the autostereogram, there are certain truths, certain categories of existing facts, that cannot be revealed by a process of pure experiment and deduction, that is, by a method of analysis. The reality of that image is there all along, and in brief moments of revelation we can catch a glimpse of it, that "other world," but in ordinary perception it is not visible at all.

Thus do I suspect, to extrapolate from this analogy, that if a spiritual world exists, it exists beyond the division of subject and object, as a superject which fuses the two in a reality greater than the sum of these parts. In contrast to the autostereogram, though, the superjective is not just an evanescent mirage-like film floating on top of reality which, perhaps just now and then, can be glimpsed for the curiosity it is. No: the superject is precisely that one "thing" that independently and actually exists. It is the world at all-inclusion, at its highest and truest level of description. Everything we see and know, everything we do or even imagine at our biological level of assimilation exists conditionally with respect to the superjective. This reality is conceived not just as a mixture of subject and object, nor even as a blending of these categories at our level of description. Rather, it is a fusion into transcendence of these categories, and that is much more significant.

I will now employ a more detailed and fruitful visual aid, with the same warning as before, that it is intended only as an imperfect analogy, not to be taken too literally. Imagine an enormous two-armed centrifuge of cosmic dimensions, as if floating in a void. Imagine also that the process of emerging from the ultimate core of the universe, and proceeding outwards through different states across ever greater "distance" from the origin, is in some way akin to the spin of this centrifuge taking hold and gradually increasing in ferocity. As this happens, the diametric arms implicit in the system begin to assert themselves and the main consequence of this spin is to separate out the original fullness into oppositely defined imbalances. In this model, by the time we arrive at the human condition, our state of being is highly polarized into extreme physicalism on the one side and a somewhat desiccated mentality on the other, which we are apt to interpret (incorrectly) as dualism. 
Alternatively, think of this centrifuge as an ice-skater. Two extreme conditions are possible for this skater. During a tight pirouette, all the energy is concentrated in a unified form at the center. This is a very powerful expression of the energy, but it is also very internalized or focused. Another way of expression exists, and that it is to divide the energy into two elements (the arms in this case) and fling them out in a centrifuge. Nothing has been lost. The angular momentum is conserved, but the energy is slowed down, and out at the extremities it takes the form of two windmilling masses.

Our mental existence is deprived of the spatial, concretizing element separated into the other principle, which carries with it the fullness of embodied presence and the bulk of causal powers. Reciprocally, brute material substance, as we call it, is deprived of the fluidity of symbol and the knowledge of consciousness. As we move away from surface experience and dreams, these principles converge again towards their apex and the fullness of being is recovered. The weightless imaginations of the dreamworld acquire a center of gravity in form, and the inertia of insensible matter recovers its connection with living presence, in an environment where consciousness and energy are shimmering facets of the same truth. This is not an unreal world; it is just the opposite. It has a higher reality index than the world we inhabit. Our world is "real" in terms of value and experience, but illusory in the sense that we imagine it to be either unconditional or final. There is no "physical world" or "mental world" except with reference to an originating or apex condition that includes them both. Centered in this apex condition, form and consciousness are coordinating aspects of each other, and so forms are perceived through consciousness.

On the centrifuge analogy, the physical world corresponds to the bipolar condition of maximum spin. Since we ourselves are wholly taken up in this "spin," as it were, we are not aware of its existence, and mistake for independent facts categories that have a reality only in terms of their opposite separator, a bit like male and female. This brings us to Twemlow's position. We increase our understanding if we lose the categories of subject and object in favor of a "dynamic tension" or "dialectic" between these categories. However, this is only half the story, for even this dialectic is an artifact of spin separation. It resembles the centripetal line of force that comes into existence across the arms of the centrifuge during separation and has only a conditional existence, like the rotating masses, like subject and object.

What really exists is not spin at all, but the "centrifuge system" in totality, which can fold inward to a state of repose or spin outward to 
a state of bipolar separation. It is this state of repose, where no spin is acting, that I propose to be the natural or irreducible ground state of the universe. The system can exist without its spin, but spin cannot exist without the system. In this state of repose the separators, which have a reality only in terms of centrifugal force, are reunited. This is the superjective, which is both immanent in, and yet transcendent of, the entire centrifugal context, the whole subject-object world with all its separating categories and dynamie tensions.

Of course, I am not suggesting that the universe is really a centrifuge. The centrifuge is a visual device conceived in terms of mechanical properties, with the particular benefits and drawbacks that brings. Defining the superjective is not easy. How do we develop a clear image of something that is larger in scope than all our possible definitions? This reality transcends subject and object. It is not a "thing," nor is it even a "mind" that perceives things. It transcends these categories: they are contained within it and expressed through it. As such it is a "category" in itself beyond categories, a multiplicityin-unity that subsumes the universe. If we are to go looking in our surface world for clues to the reality of the superject, we must search for the underlying and the abiding. I suggest that there are three properties of our world in particular, known to our experience, which resist being further broken down or reduced.

The first of these properties is a certain abstract lawfulness underlying the material forms of the universe. Material forms themselves do not qualify: they can be destroyed, and in the end destroy themselves. However, if we search back far enough we begin to discover suggestions of a deeper order in the world, one that does not depend upon perishable laws, but upon "natural laws" that are somehow abstract and eternal. It is the mathematical understructure of reality. This order is more subtle than it may at first appear, though. It is not a rigid edifice, but somehow expresses from within itself a genuine and fundamental "openness" in the structure of events, quantum possibilities, a free element that cannot be further reduced.

The second clue is the seemingly nonlocal nature of consciousness, even within the context of the brain itself, without even mentioning the phenomena of near-death experiences. This is reflected also in the nonlocality of quantum processes. "Parts" of a system supposedly separated in space and time react with an immediate and infallible "knowing" of each other's activities. The chances of such a striking fact not being deeply interwoven with the root potentials of conscious awareness are slim indeed. Thus even in the world's most elementary 
Figure 4

The Superjective as the Fulcrum of Reality, with Form, Consciousness, and Energy Spun out from the Center.

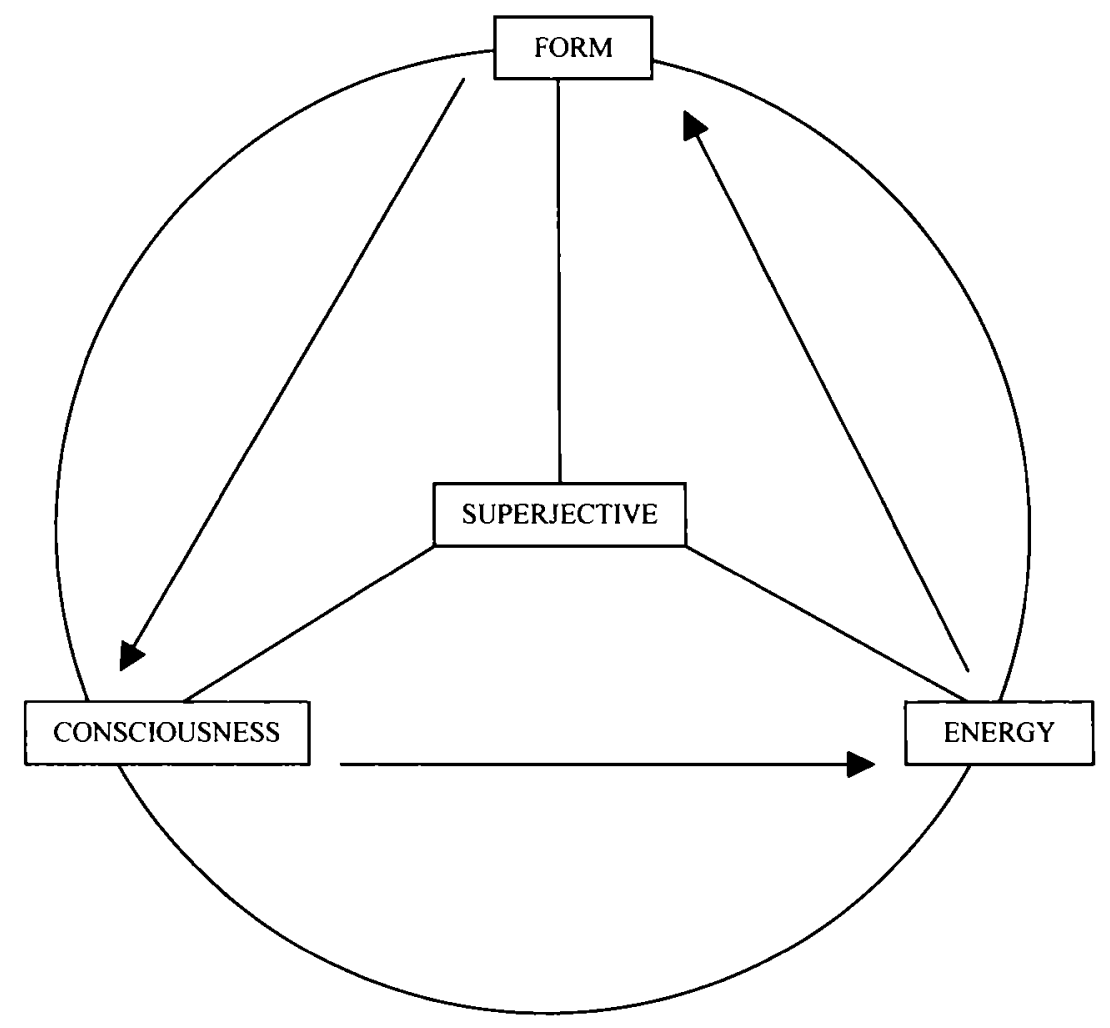

systems of expression there exists the rumor of an indelible and irreducible consciousness, defined simply as a system's awareness of its aspects in nonlocal relation.

Finally, there is the particular indwelling property of existing things we refer to as energy, the actualizing power or expressive dynamism of being. This also resists further reduction.

I will now suggest that the superjective has these three particular aspects that contribute to its character. This is already a certain level of fallacy. As soon as we assign components to the superject we are already moving away from the truth of what it is. It is a wholeness-inone. It does not and cannot have component "parts." In Figure 4 the 
superjective is depicted as occupying the absolute center or fulcrum of reality, but as being capable of throwing out from itself these three almost-autonomous aspects, in the spirit of the centrifuge model. These outward projections are like shadow domains (in the Platonic sense), or sub-level syntheses of its reality. For the superject itself, centered at the fulcrum, existence is a completed transcendence of these three aspects. It is a reality capable of knowing itself, and that knowing is a totality or consciousness; it is a reality embodying an inherent lawful order underlying the cosmos; and it is a reality capable of full and free action, limited only by an inability to contradict its own inherent order. In the pristine state, this triad exists in perfect harmony. Consciousness, law, and immanent power are perfectly measured each to the other. In some mysterious way, however, these three aspects are also capable of acting $a s$ if they are separate. If I am asked how this is so, or exactly how this is achieved, or exactly what "centrifugal spin" corresponds to in the real situation, I must candidly admit that I have no clear idea. Nevertheless, $I$ think it is possible to make some tentative remarks.

As soon as we move away from the fulcrum point on the diagram, we emerge spontaneously from the superject reality system. The condition of all-inclusion is lost, and the subject-object dichotomy comes into play. On our analogy, the perfect balance of the triad resonance is broken. The three shadow domains together make up the world we know, the world in which we live from day to day, and yet the irony is that the superject (and the superjective in ourselves) permeates through and through. It is immanent in and yet transcendent of the entire process-structure, like the image in the autostereogram. The three shadow domains represent certain limiting extremes or states of maximum abstraction from the superject reality system. Consider each of these as one element in the triad expression of "worldstuff" pushed to its totality at the expense of the others.

Thus, if "law" is pushed to its totality, the other factors (consciousness and immanent power) are maximally damped. From the subtler reality of the abstract order, existing in harmonized relation with the other two aspects, comes boiling up the derivative existence of a more rigid and concrete order, which nonetheless is generated by, and according to the laws of, the origin's inherent lawfulness. The end result is a system of order in which consciousness is present at a bare minimum (therefore "unknowing" or blind process governs the situation) and immanent power is present at bare minimum (therefore rigidity and implacable local forces act within it). It is an imperfect 
abstraction from the origin's lawfulness, the forming principle precursing matter.

As this lawfulness emerges from the original or superjective state, by suppression of the other two principles, it is rendered imperfect with respect to what is unconditionally "real," because only the superjective is unconditionally real. All the while, as this transformation moves it further from the condition described at the origin, its potency decreases. It is therefore only an approximation of the origin's lawfulness, for that lawfulness can only function perfectly in the superject condition described by completed transcendence with the other two principles. This approximation somehow takes law out of its abstract (subtle) order and gives to it the potential for what we call manifestation, while at the same time incurring entropy. Forcing this out further and further we approach an unattainable asymptote defined by complete lawlessness or total entropy. It there becomes mere chance or aggressive randomness at loose in the world. In this form it can destroy mind and order together.

Likewise, if the immanent power is pushed to its totality, consciousness and law are maximally damped. This is the immanent power in the stepped down form of material energy, uninformed by any state of consciousness, and abstracted from lawfulness. In this scenario it can be the engine of either order or entropy. All the while, moving away from the superjective, its potency decreases. If this separation is pushed to its extreme, existing forms are further and further de-energized until the result is a "frozen" rigidity of structure or the inability of structure even to form in the first place.

Finally consciousness, if pushed to its self-isolated totality, loses all the lawfulness and immanent power that inform it in the superjective reality system. All the while its potency decreases. It becomes unconsciousness at its nadir, the worldstuff in its state of maximum unrealization. These three components: rigid material structure, chaotic processes, and unconsciousness, describe the dominant forces in the early beginnings of the universe, since together they represent the condition of maximum abstraction from the superject reality system.

The three shadow domains indicated can never proceed to absolute elimination of the other two. Within this system, there is no such thing as order without consciousness and immanent power at least residual or implicit within it. These end points are arrived at by transformational elasticity and they are not Cartesian separations. Each contains the imprint or "memory" of the others within itself; that is to say, they are monadically embedded. 
In Figure 4, I have placed the superjective state (or divine reality) in the center, with all three derivative states circling around it like satellites in a constant state of motion. This evolutionary racetrack consists of the worlds of consciousness, form, and energy respectively, each finally isomorphic with the other. Left to their own devices, due to their monadic embedding, patterns in any one will begin to combine in new configurations that express the other principles from their latent state.

It makes little difference where on the diagram we choose to start, but let us take "form" as the beginning condition. This is the approximation to the origin's lawful order, which gives rise to the potential for that much cruder order we know as physical manifestation. These approximate laws begin to combine in ways which reexpress from within themselves the dynamic aspect or immanent power. As this happens, "form" gives to the immanent power (energy) the structure of lawfulness, although imperfect, while energy gives to form the actualizing force of manifestation.

These material processes ("inanimate world") then continue round the evolutionary racetrack and sooner or later combine into forms which begin to re-express within themselves an imperfect image of the origin's consciousness in the form of biological awareness. Why? Evolution actually is the process of the implied re-emerging into expression by creative recombination. Material form now gives to consciousness the capability of expression in a biological context, while consciousness gives to material form the inner character of selfawareness, a consciously realized (as opposed to an unconsciously realized) purpose, and an integrating or top-down organizing influence on the material state.

Nor does it end there. It continues on round the circle. Consciousness, through patterns imbued with dynamic potency and enabled by material form, begins to combine and express from itself (imperfect) versions of the abstract order again: thoughts, imaginations, deeds, rituals, religious impulse, art, and so on. However, these "tend towards" an absolute defined by the origin, which also retains a weak direct link with each of the three worlds described by consciousness, form, and energy. For consciousness, this residual contact with the divine wisdom manifests as insight, inspiration, mathematical genius, moral intuition, spiritual intuition, and the sense of beauty. Although most of these manifestations relate to order, the tremendous sense of energetic power in spiritual experiences like the NDE arises from consciousness intuiting or coming into the ambience of the origin's 
immanent force. Also in terms of the NDE, the "vision of knowledge" equates to a direct apprehension of the origin's universal information structure or inherent lawfulness.

In the triangular isomorphism of consciousness, form, and energy, we presently have a formal description in terms of one side only: Einstein's equality of matter and energy. I suggest that the isomorphism of consciousness and form is precisely why mental insights commute with the world of "natural law." I further suggest that the isomorphism of consciousness and energy is precisely why the world of perceptions commutes with the world of material objects; and that the isomorphism of form and energy is precisely why the world of mathematical abstractions commutes with the world of material objects.

\section{Seven Patterns Suggestive of Superjective Ontology}

Theories that claim some basis in fact and are not just fond wishes of their originator should be expected to hold some predictive power. Superjective ontology has certain consequences that tend to follow naturally from its defining axioms. I will now elaborate on seven patterns that I believe are strongly suggestive of the position laid down in the previous section.

Much of this will relate directly to so-called "paranormal" phenomena, something which may irritate critics from the outset. This is no accident. In consequence of the premise that our categories are incompletely assimilated or misdescribed, there comes into existence in our experienced world, of necessity, a "fault line" where the ontological violation concentrates. This is no ordinary problem, however. Try as we may, at times to absurd or heroic effort, the fault line asserts by demonstration that it cannot and will not be eradicated. It consists of the ultimately challenging paranormal precisely because the underlying problem is fundamental, residing as it does in an ontological error that can never be remedied by yet another hypothesis, however ingeniously conceived, in the same old mold.

Pattern \#1: Paranormal phenomena display superject-structured modality. Objects become imbued with "subjectivity." A psychic picks up an innocuous item once associated with a murder and reads from it an entire history of emotion. Again, it need not concern us here to validate the claim; it is the nature of the claim itself that is of interest. Haunted houses exude an "atmosphere." The world itself 
demonstrates "synchronicity." Reciprocally, there is an externalization of mental realities. The ecstasies of Christian stigmatics imprint themselves in the flesh, and the mind has psychosomatic influence over the body. People who witness unidentified flying objects (UFOs) experience elaborate events with their roots in human mythology, and yet there are scorched branches and uncorrelated targets on radar. Images of deceased relatives turn up mysteriously in photographs. The mind influences the motion of objects by psychokinesis. Adolescent tensions unleash a poltergeist. Violent or harrowing circumstances "imprint" themselves upon a locale by sheer force of emotion.

There is a distinctive signature here. On our hypothesis, we may say that these phenomena are nothing less than fragmentary and momentary glimpses of the transcending superject within our subject-object world, a flash of the cosmic autostereogram behind the concealing veils of random pattern, metaphorically speaking.

Pattern \#2: When undue (ontological) pressure is exerted either on the category "objective" or the category "subjective," the overcategory begins to appear. There are several demonstrations of this. In the near-death experience, it matters little whether the precipitating stress acts on physiology or psychology (as in so-called "fear-death experiences"): the resulting visionary pattern is almost identical. Many theories involving biological and psychological defense mechanisms have been advanced, but intriguing as these theories may be, I suggest that the real cause is yet more radical than any of us have supposed.

By nature of the argument, subjective and objective are in the last analysis partial or unreal descriptions; that is, they are subontological. Another way to look at it would be to say that either of these categories can take only so much "reality stress" before its illusion fails. This applies to the world of matter and the world of mind in equal measure. Chase too far into either of these realms, as if you believe that it alone exists, and the superjective asserts itself.

A good example is meditation, where the individual emphasizes indwelling of consciousness and the world of mind to the point where appearances can no longer be sustained and the world begins to encroach as it really is. All the principal mysticisms warn that paranormal phenomena can manifest under these conditions, if only so that they can be avoided on the spiritual path.

Moving in the opposite direction, as we pursue matter down to its elementary particles and beyond, we suddenly find ourselves in a quantum realm, where the objective nature of the world becomes 
a shadowy dance of phantoms, and behaviors characteristic of the overcategory, such as nonlocality in space and nonspecificity in time, begin to appear. A mysterious "influence of the observer" also enters the picture. I will again ask you to notice the same distinctive signature emerging from these facts.

Pattern \#3: Consciousness and energy are isomorphic. This is one of the more important conclusions to be drawn from superjective ontology. Energy and form are separated into one category, in which nonetheless a residuum of consciousness capable of driving evolution at unconscious levels and a nonlocal spatial information patterning are monadically embedded. In the opposing category, consciousness and nonlocal information are maximal, while form and energetic immanence are monadically embedded. Paranormal phenomena in which conscious will is transmuted to energy include psychokinesis and healing. Phenomena in which structures of energy are transmuted to consciousness include dowsing and kundalini arousal.

Pattern \#4: Immersion in the superjective expresses an aftermath radiating oppositely into consciousness and energy. For example, Kenneth Ring (1992) described a psychophysical cluster of aftereffects to NDEs and UFO encounters. Although much work remains to be done on the electromagnetic interference effect and the inability to wear wristwatches suggested by his research, this pattern has long been a simmering rumor in witnesses to UFO-like events. Examining Ring's data, we find on the subjective side, changes in consciousness, including mood swings, paranormal events, and a sense of mental expansion; while on the objective side we have a reduced sleep requirement, altered metabolic processes, increased sensitivity to physical stimuli, a sensation of "energy currents," and electromagnetic interference. The main point to be digested here is that this scatter of seemingly diverse phenomena is in fact constellated, having a natural and immediate explanation on the present perspective. If the NDEr is immersed in the superjective (even briefly), where the potency of consciousness-energy is much greater than in the physical context, he or she becomes impressed with this greater potency and its aftermath forks like a lightning flash into the sub-ontological categories of mind and body during "reentry" from the near-death experience. Both apparent categories are put under a stress to absorb or adapt to the greater reality index signified by the visionary near-death experience.

Stigmata do not feature significantly in NDEs, although I would suspect that as more frightening experiences are studied, this pattern 
too will be disclosed. In the study of NDEs in India by Satwant Pasricha and Ian Stevenson (1986), one gentleman (Durga Jatav) claimed that fissures had appeared on his knees subsequent to an unusual leg-severing ordeal during his experience (Pasricha and Stevenson, 1986); and in the Vision of Fursa, described by Carol Zaleski (1987), the visionary retained a permanent lesion around the neck and jaw, where a flaming soul of the damned was said to have been hurled in his direction by infernal being.

In major UFO cases, there is also evidence that the primary event precipitates simultaneously into the subjective and objective realms of the witnesses, in a way so deeply interwoven that we are left grasping for an explanation. In the various attempts to account for this uncomfortable fact, some theorists (Persinger, 1977) have postulated an object-to-subject causal chain, where a plasmic blob or energetic column in the landscape influences the mind through the temporal lobes and thus distorts it; while others (Devereux, 1989; Randles, 1990) have argued for a subject-to-object causal chain, where a hidden talent of the mind is capable of reaching out with a psychokinetic influence and morphing the plasmic mass into approximate forms. Each of these is an innovative and courageous idea, but I believe that they all reflect the same chronic malaise. When the first sentence is being laid down, the critical error has already been made, and the structure of the thesis itself guarantees that the solution is unattainable. There is no causal chain. The origin of the event is in the superjective, and local ontology is violated in the presence of the phenomenon.

Pattern \#5: Immersion in the superjective fuses subject and object, consciousness and energy. If close attention is paid to the statements of near-death experiencers, this pattern becomes evident. Moving towards the superjective, a structured mental creation (or presence) must also express the primary immanence we call energy in direct proportion to its integrity, power, and organizing force. Reciprocally, the more energy you have, the more closely you approach a sentient origin of all consciousness and being, by whatever name we call it.

Consider first the tunnel experience. Is this a mental symbol or a conduit of energy? Hold determinedly to either position and we are forced to ignore certain important testimony from NDErs. In different experiences, one or the other interpretation may appear to predominate, but neither is sufficient for a complete understanding of all the features of tunnels. In cases where patterned ideation appears to gain the upper hand, the experiencer may float along a tunnel made of 
steel panels riveted together, sail through a "cave" in a small "boat," or walk down a "corridor" perhaps containing windows and doors. Where structured energy predominates, the experiencer senses the presence of overwhelming power, infinite acceleration, immeasurable distance, and has the impression of being surrounded by a protective boundary.

In the superjective domain, the rules have changed. When you now bring into existence any mentally patterned imagination, you are also creating a structure of energy. When you are now exposed to currents of energy, they are assimilated reflexively as "sensory" experiences and structured imagination. As consciousness phases deeper into the superjective, these behaviors merge into one and the same event. The phenomena of the NDE are not watery symbols only, but ideoplastic structures in imagination as energy. Furthermore, if consciousness at this depth equates to energy, then consciousness itself will be envisioned in terms of energy, and a stronger source of consciousness relative to yourself will be experienced as an intensely luminous "radiance" of light, warmth, and power.

There are some additional clues. The "libraries" of this other world do not just contain knowledge, they are "made of" knowledge. We have grown accustomed to the idea, perhaps to the reference, but what does it mean? Then there is the sense, often reported, that the environment in which NDErs find themselves is somehow itself a presence. Running through all of this is an intense struggle to fit the round peg of an extraordinary event, standing outside our normal taxonomies of experience, into the square hole of the divisions of classification appropriate to ordinary consciousness, using a framework of language that itself evolved to communicate and reinforce those divisions.

Consider again the "silver cord" sometimes reported in out-of-body experience (OBE). Applying the "objective" argument, we would be forced to conclude that it was just a mental symbol and that it never was real. Alas, this explanation does not satisfy people who have had a very real and "physical" experience of this cord. Now make the shift to superjective understanding. Consciousness and energy are isomorphic, so if the body is still alive, there will remain a link to the experiencing mind, which has features descriptive of both. Trends in the patterned imagination of the experiencer will cast this link in one or another concrete form, which has full superjective validity the moment it is created. Nowhere in this reality is there an abstract symbol or an inanimate thing. As you think, so changes energy. As energy expresses in pattern, so it appears in experience. Much the same argument applies to the astral body, sacred presences, 
impassable boundaries, eschatological landscapes, chakras, kundalini, and flying saucers.

In Carol Schorah's NDE, she distinctly felt being "sucked" back into her body (a sensation reported in numerous OBEs), with the last part happening very quickly (Ritchie, 1994, p. 149). John Priest felt himself pulled upwards by an incredibly powerful force, as if on a great elastic band (Ritchie, 1994, p. 131). Helen Nelson was wrenched back into her body with a rushing sensation, an excess of electrical energy, and a "bang" (Taylor, 1981). Anne Allcott seemed to "pour" back into her body (Fenwick and Fenwick, 1995, p. 27). Peter Thompson "slammed" back in (Fenwick and Fenwick, 1995, p. 6). Ivy Davey underwent a series of "shudders" followed by a "click" (Fenwick and Fenwick, 1995, p. 41). S. Woodham was pulled back by an implausibly long "nightdress" (Fenwick and Fenwick, 1995, p. 78). Ring (1980, p. 100) recorded the testimony of a man who experienced falling back to his body with a jolting impact, and another subject who experienced being pulled out of a huge vacuum surrounded by thunderstorms, feeling (rather like Sylvan Muldoon) as if his body was being torn apart. Just when did our mere fantasies take on the properties of fluid dynamics, elastic rebound, and charge density?

Thus the light of the NDE is a light that would "immediately blind you" if seen with the physical eyes. Travelers through the tunnel experience "tremendous energy." The being of light is not only a being and not only a light, but also a devastating source of energy. God is perceived as the repository of all energy in the universe. It seems quite clear to me that NDErs are not just being "poetic" when they report these things: they are thoroughly in earnest.

It is important to stress that we are not talking about physically described facts. Any such facts could only be (as we call it) objective in character, and thus trapped on the dichotomous side of the superjective's "event horizon." There may conceivably be discernible consequences in physical patterns of certain superjective events, as noted in \#4 above, but that is really a different question. This is the tenacious error of concretism concealed in the astral body doctrine and in the equally misdiagnosed "problem" that an NDEr out of his or her body would need to have physical eyes that absorb photons. As soon as the categories of mind and energy begin their implosion towards each other, the transformational vector leading to the superjective appears, and from that moment on all our corporeal talk about "energies" and "imaginations" bears but the crudest approximation to the events in process. 
Pattern \#6: Paranormal phenomena manifest where the interjective (subject-object) description is simplest. In Cartesian terms, there is no essential difference between two people standing in a field and a hundred people standing in a field. Nothing in the structure of observers or landscape is altered by such multiplication. This relationship does not hold in superjective ontology. The total reality description of a hundred people standing in a field is something much more complicated than that of two people standing in a field, and even that of two people is considerably more complicated than that of one alone. Why? Simply put: subjective presences contribute to the stability and integrity of the total reality envelope in which they participate, and are not just passive observers.

Now I am not claiming that the difference is large. Nor am I saying that the field vapors away into unordered chaos when it is not being observed. What I am saying is that the human psyche is an authentic contributor to any reality state in which, at the moment in question, it is a component part; and that the combination of one human psyche and an isolated environment gives the simplest of all descriptions. If that single person happens to have unusual mental powers or be what we might call "psychic," the total reality description in which he or she is participating may be significantly altered. Therefore, in contrast to the materialist prediction, the full reality envelope consisting of "this person plus this environment" is not necessarily the same thing at all as the reality envelope described by "some other person plus this environment."

The condition in which paranormal phenomena are likely to manifest is precisely this case of one observer in dialogue with the environment, especially an individual already sensitive to (or conducive to the expression of) superjective potentials. In that scenario there is the maximum leeway for incursion of the superjective into the subject-object world. As soon as additional persons are added to the scene, the total reality description rapidly becomes more complex. The majority of paranormal events claimed in the physical context involve just one or two people. A small proportion involve a handful. Very few indeed involve more than a handful, and as a general rule, the more observers are present, the simpler and more ambiguous the phenomena become (this is related to \#7 below).

Each of these contributing psyches may have different capabilities for potentiating the superjective, different aptitudes for sensing its incursion, and different thresholds of tolerance to its presence. Thus as more observers are added, the likelihood of a superjective event 
shrinks. Furthermore, what one witness to a superjective event perceives may be approximately constrained to (but not identical with) what another witness perceives, because each psyche is experiencing through its own legitimate roots in the total reality state.

In the otherworldly phase of the $\mathrm{NDE}$, the natural conclusion must be that consciousness is entirely immersed in the superjective reality system (although, it would seem, with varying degrees of completeness). As it "returns" from the experience, it emerges once more into what I refer to as interjective duality. However, even this cannot be assumed, in wake of the NDE, to continue simply as it was before. It is quite possible that the experiencer's ontological embedding may also be reset in aftermath of the experience, and that in some cases this extends even to the world or "reality envelope" inhabited by that subject. Although such things as waves of inexplicable synchronicities, haunting type phenomena, or poltergeist-like outbreaks are not a predictable outcome of NDEs, they have been informally reported often enough to raise the suspicion. In the terms stated above, the interjective context inhabited by the subject has undergone a change. It is as if the superjective leaves something of itself in the voyager, or as if the entire organism, once exposed, retains always thereafter the indelible "memory" of the unconditionally real.

Pattern \#7: Paranormal phenomena are a paradox that will always resist consummation in a Cartesian universe. A century of psychical research, and in recent decades parapsychology, has failed to advance our understanding of these events to any significant degree. This becomes painfully obvious when we compare its successes with such disciplines as medicine or aeronautical engineering. While giant strides have been taken in these areas, intractable arguments about the very existence of telepathy and psychokinesis can still be found today. Despite this, and despite an equally long century of enthusiastic debunking by those who believe that such terms belong to the argot of the poorly educated, the phenomena refuse to go away. Nor can they go away, because they stand for certain ineradicable behaviors of the "real world."

The clue to the problem has already been given. Medicine and aeronautics, like most of science, take as reality the authentic existence of objective and subjective categories. They actually consist of thought-and-action structures built out of those categories, so it comes as no surprise that in terms of those descriptions they have been very successful. It takes a moment to see this clearly. 
Please understand: I do not claim that "subjective" and "objective" are entirely unreal or mere arbitrary illusions. On the contrary, they are authentic sub-ontological forms deriving from the superjective. In pursuing their patterns it is possible to gain real insight, even some insight into the superjective itself, but there exists a kind of asymptotic limit to what can be achieved in this way.

Until very recently, human science has been indelibly bonded to the Cartesian assumption. We praise the "objective," with some justification. We denigrate the "subjective," with less justification. We demand proof, not realizing that in the very structure of the demand there appears a "proved" and a "prover." This approach to the world was a major advance over medieval superstitions, and it is in the nature of most scientists to believe that the future will consist of better and better science, and not (as is much more likely) of something that will be as different again over science as science was over the mediaeval worldview.

Paranormal phenomena operate in superject-structured modality. Thus, when we go in search of a mysterious "influence" acting upon the mind, or a mysterious "force" acting from the mind, we have already ensured that it will never be found. This error is reinforced and perpetuated in an endless stream of new scientific theories aspiring to a unified explanation of the unexplained on the basis of some recently discovered panacea; and the error is reinforced in the very terminology we apply to the challenge: "telepathy," a "sixth sense," and "mind over matter." Subject and object are the twin shadows of the superjective, so anything of the superjective that we could find :- those categories could itself only be a derivative phenomenon or symptom that would evaporate on close inspection.

Superjective events can manifest in the world in one of two ways. The first is by appearing to move among the patterns of subject and object, as weird and fragmentary phenomena that defy explanation at our level of synthesis. Moreover, they cannot have an explanation at our level of synthesis. The second is a full scale ontological rupture, as outlined in \#4 above, where local events are reconstellated to a superjective description. Nor is this the worst of it, for in the unshielded presence of true reality, the continued existence of the under-real, world and mind, perceived and perceiver, is all at once imperiled.

This has significant implications for terrifying NDEs, where the individual insists on the continued existence of an ego structure (and hence a fully crystallized subject-object dichotomy) in the face of the superjective reality system. I agree with Ring that resistance to 
surrendering the ego is a very important factor, but what I must bring out here is the critical role of the ontological dimension in evoking the existential terror. We can see now that what the experiencer is really doing is "arguing" for the existence of unreal categories in the immediate ambiance of the real, and the result can only be the terror of an ever-imminent annihilation. I also believe that the visceral level of fear endured by many UFO witnesses extends from the same cause, and that the experience processes itself into cycles of imagery that try to make sense of this appalling emotion, without parallel in ordinary human experience. There is a lot more to this issue than I can summarize here, and I have developed a more complex argument elsewhere (Carmen, 1998).

We will never have either an explanation or a demonstration of paranormal phenomena in terms of scientific methodology as presently described. They will always conceal themselves in ambiguity, because their consummation by formal proof in a subject-object universe is a fundamental paradox. The real will never be verified or contained in the terms of the under-real. What we have are astonishing phenomena taking place when the opportunity for formal measurement is momentarily absent: the poltergeist launching a dish from its shelf when the investigators are changing the film in their camera; the NDEr accurately perceiving events in another ward of the hospital, but never in an observational context that would enable us to understand how it was done. It is true that impressive results have been obtained in a few parapsychological protocols, for example Robert Jahn's experiments investigating the mind's influence on large statistical cascades, but these are always under circumstances where the actual effect in question is "far from direct observation," safely embedded in quantum events or enormous number strings, and so the conditions described under \#2 are satisfied.

\section{Conclusion}

I am not about to lose sight of the fact that the superjective is a speculation. But in case we should now conclude that the entire project is hopeless, or that we are doomed never to make any real progress in the understanding of the near-death experience or other superjective phenomena, let me offer some encouragement. The barrier in question is not erected against an understanding of the superjective itself, nor even against the possibility of something akin 
to an applied "science" in this domain, but against the possibility of achieving such an understanding while we cling to methodologies and conceptual structures predicated upon ultimately nonexistent categories. Nor do I entirely rule out the possibility, after Ring, that the "universe" is deliberately drawing these seemingly impossible facts to our attention in a ruthless and systematic manner, precisely because they represent the next quantum leap necessary in our understanding.

I accept the fact that in certain respects my position may seem very uncompromising. Understood correctly, its succussive force on the great bulk of our theorizing is irreparably violent, and by no means limited to the field of near-death studies. Nevertheless, I stand by my prediction that any less decisive a stroke will eventually be found wanting, and that the real choice concerns the possibility of realizing this sooner, or being forced to realize it later, after many bitter (and unfathomably bewildering) disappointments.

Furthermore, so long as those actually sympathetic to spiritual experience continue to argue for the present limitations, so the neardeath experience will always seem to be at risk from yet another theorist with yet another model, and yet more certainty that he or she has cracked the code of the NDE. In truth, there is no code. The superjective has no cipher but itself. However, it is much worse even than this. There are no "temporal lobes" either; there is no "ketamine"; no "dying brain"; no "Darwinian mechanisms"; nor is there even any "dissociation," "hallucination," "positive affect," or "ego defense." All of these things, including their previous incarnations and yet-to-behypothesized equivalents, fail to have an existence in anything like the form we ascribe to them.

Finally, I am not attempting to diminish the divine by describing it in the ultimately technical terms of a "superjective," or as anything else. I have written nothing in this paper of the overwhelming love many near-death experiencers have reported. This is not because I believe it to be unimportant (far from it), but because the present study has focused its beam in the somewhat narrow realm of ontological inquiry. The ultimate is not this. The ultimate is simply at least this.

\section{References}

Arnette, J. K. (1992). On the mind/body problem: The theory of essence. Journal of NearDeath Studies, 11, 5-18.

Arnette, J. K. (1995). The theory of essence II. An electromagnetic-quantum mechanical model of interactionism. Journal of Near-Death Studies, 14, 77-99. 
Becker, C. B. (1995). A philosopher's view of near-death research. Journal of Near-Death Studies, 14, 17-27.

Blackmore, S. J., and Troscianko, T. S. (1989). The physiology of the tunnel. Journal of Near-Death Studies, 8, 15-28.

Blackmore, S. (1993). Dying to live: Near-death experiences. Buffalo, NY: Prometheus

Carmen, A. A. (1998). Rhythms of the overworld. Edinburgh, Scotland: Sushumna Vision.

Crookall, R. (1960). The study and practice of astral projection. London, England: Aquarian Press.

Crookall, R. (1964). More astral projections. London, England: Aquarian Press.

Crookall, R. (1980). Casebook of astral projectión. Secaucus, NJ: Citadel.

Descartes, R. (1983). Principia philosophiae. Dordrecht, Holland: Kluwer. (Original work published in Latin in 1644)

Descartes, R. (1990). Meditationes de prima philosophia. Notre Dame, IN: University of Notre Dame Press. (Original work published in Latin in 1641)

Descartes, R. (1994). Discours de la méthode. Notre Dame, IN: University of Notre Dame Press. (Original work published in French in 1637)

Devereux, P. (1989). Earth lights revelation. UFOs and mystery lightform phenomena: The Earth's secret energy force. London, England: Blandford Press.

Dutheil, B., and Dutheil, R. (1990, Spring). Towards a superluminous model of consciousness. IANDS-UK News Bulletin.

Fenwick, P., and Fenwick, E. (1995). The truth in the light. London, England: Hodder Headline.

Gómez-Jeria, J. S., and Saavedra-Aguilar, J. C. (1989). A neurobiological model for neardeath experiences. Journal of Near-Death Studies, 7, 205-222.

Gómez-Jeria, J. S., and Saavedra-Aguilar, J. C. (1994). A neurobiological model for neardeath experiences. II: The problem of recall of real events. Journal of Near-Death Studies, 13, 81-89.

Gómez-Jeria, J. S., and Madrid-Aliste, C. (1996). Evolution and the relationship between brain and mind states. Journal of Near-Death Studies, 14, 251-272.

Kazanis, D. (1995). The physical basis of subtle bodies and near-death experiences. Journal of Near-Death Studies, 14, 101-116.

Lawson, A. H. (1994). Response to the Twemlow paper [Letter]. Journal of Near-Death Studies, 12, 245-265.

Leibniz, G. W. (1965). Monadology. Indianapolis. IN: Bobbs-Merrill. (Original work published in German in 1714 and published as Principia philosophiae in Latin in 1721)

Muldoon, S., and Carrington, H. (1929). The projection of the astral body. London, England: Rider and Company.

Muldoon, S., and Carrington, H. (1951). The phenomena of astral projection. London, England: Rider and Company.

Noyes, R., and Kletti, R. (1976). Depersonalization in the face of life-threatening danger: A description. Psychiatry, 39, 19-27.

Ouspensky, P. D. (1931). A new model of the universe. London, England: Routledge and Kegan Paul.

Persinger, M. A., and Lafrenière, G. L. (1977). Space-time transients and unusual events. Chicago, IL: Nelson-Hall.

Persinger, M. A. (1983). Religious and mystical experiences as artifacts of temporal lobe function: A general hypothesis. Perceptual and Motor Skills, 57, 1255-1262.

Pribram, K. (1979, February). Holographic memory. Psychology Today, pp. 70-84.

Randles, J. (1990). Mind monsters. Wellingborough, England: Aquarian Press.

Ring, K. (1980). Life at death: A scientific investigation of the near-death experience. New York, NY: Coward, McCann, and Geoghegan. 
Ring, K., and Rosing, C. J. (1990). The Omega Project: An empirical study of the NDEprone personality. Journal of Near-Death Studies, 8, 211-239.

Ring, K. (1992). The Omega Project: Near-death experiences, UFO encounters, and mind at large. New York, NY: William Morrow.

Ritchie, J. (1994). Death's door: True stories of near-death experiences. New York, NY: Dell.

Sagan, C. (1979). Broca's brain: Reflections on the romance of science. New York, NY: Random House.

Siegel, R. K. (1981, January). Accounting for "afterlife" experiences. Psychology Today, pp. 65-75.

Talbot, M. (1991). The holographic universe. New York, NY: HarperCollins.

Taylor, D. (1981). Profile of an experiencer: Helen Nelson. Vital Signs, 1(2), 5-6 and 14.

Thaler, S. L. (1995). Death of a gedanken creature. Journal of Near-Death Studies, 13, 149-165.

Thaler, S. L. (1996). The death dream and near-death Darwinism. Journal of NearDeath Studies, 15, 25-40.

Twemlow, S. W. (1994). Misidentified flying objects? An integrated psychodynamic perspective on near-death experiences and UFO abductions. Journal of Near-Death Studies, 12, 205-223.

Zaleski, C. (1987). Otherworld journeys: Acccounts of near-death experience in medieval and modern times. New York, NY: Oxford University Press. 